\title{
ELF Extended Low Frequency Sensor Designs
}

\author{
Kevin Westhora \\ ${ }^{1}$ Dytran Instruments Inc., Chatsworth, CA, U.S.A
}

\begin{abstract}
ELF Extended Low Frequency Sensor Designs:
Piezoelectric accelerometers are dynamic sensors with a usable frequency response that goes from fractions of one Hertz over $10 \mathrm{kHz}$. A piezoelectric sensor can de designed with a very low frequency response, called quasi-static, but it won't be a true static [DC] response.
\end{abstract}

DC type Accelerometers that can measure from static, zero Hertz; up to a few thousands Hertz, but not more.

The need for a single sensor package that can cover the complete frequency spectrum response from zero Hertz to more than $10 \mathrm{kHz}$ convinced Dytran Instruments to develop the ELF which merges two sense elements combined into one output..

Key words: piezoelectric, vibration, accelerometers, MEMS, variable capacitance, Extended Low Frequency, DC, $10 \mathrm{kHz}, 15 \mathrm{kHz}$.

\section{Piezoelectric Accelerometers}

Piezoelectric accelerometers are dynamic sensors. Their frequency response goes from fractions of one Hertz, up to the natural resonance frequency of the sensor element. Accelerometer users will utilize only the portion for the frequency response (in which the signal!) that it is proportional to the acceleration to be measured. Therefore, the usable frequency response, typically, will go from a few Hertz to about $1 / 3$ of the natural frequency of the element, that usually goes to higher than $10 \mathrm{kHz}$. A piezoelectric sensor can de designed with a very low frequency response, called quasi-static, but it won't be a true static [DC] response. This fact of the dynamic behavior of all piezoelectric sensors is well known, and an accepted limitation by the users of this type of sensors..

\section{DC-Type Accelerometers}

Accelerometers that can measure static signals are called DC types. They can measure from static, zero Hertz; up to a few thousands Hertz. DC types sensors are using different technologies on the sensing element, some are
MEMS, with a Silicon element in cantilever with a seismic mass of the same material, with variable capacitance sensing, servo [force balance], or strain gauges, also implemented in MEMS devices.

\section{Using both types instead of choosing}

Dytran instruments offers both types of sensors, piezoelectric accelerometers and DC MEMS accelerometers. In typical applications, a customer is only interested in a portion for the frequency response of the sensor, and more specifically only certain frequencies of interest. However, in occasions where a customer requires both types of signals, or a full frequency spectrum of the signals, they must use two different sensors, one DC MEMS type, with low frequency response, and a second one, piezoelectric type with high frequency response, then apply a low pass filter on the DC accelerometer with operating bandwidth from zero to a few hundred Hertz, and a high pass filter to the piezoelectric sensor that operates from just below $100 \mathrm{~Hz}$ up to its maximum operational bandwidth, usually $10 \mathrm{kHz}$. The data collected will be processed to obtain the full frequency spectrum of the vibration on the application. 


\section{Accelerometer Testing Challenges}

There is a need for a single sensor package that can cover the complete frequency spectrum response from zero Hertz up to 10 $\mathrm{kHz}$. In the past, users of the low frequency response, and other inertial devices, DC to 100 $\mathrm{Hz}$, found that high frequency vibration signals in other orthogonal axes, than the axis of interest, result in a non vibrational displacement [sculling motion] within the operational bandwidth of the DC low frequency accelerometer. There are several well known algorithms used to compensate for sculling and pseudo sculling motions, but these add to the complexity of the data acquisition and analysis. Furthermore, they need the input of the secondary sensor measuring the magnitude of these high frequency vibrational signals to apply the proper compensation or correction values.

\section{Dytran meets the Challenge}

The need for such single sensor that can cover the full frequency response spectrum convinced Dytran Instruments of pursuing such endeavor. These are the characteristics and operational principles of the ELF accelerometer Dytran model 7705A [Extended Low Frequency accelerometer].

The main principle of operation of Dytran model $7705 \mathrm{~A}$ is the instrumentation amplifier at the output buffer. The positive input is provided by the variable capacitance element and the negative input is provided by the piezoelectric element. Both variable capacitance element and piezoelectric element are filtered appropriately (low pass single pole and high pass single pole respectively) to provide the corner frequency of both filters precisely matched at approximately $30 \mathrm{~Hz}$. Both signals are also out of phase as they enter the instrumentation buffer

\section{Sensor options}

Dytran model 7705A will be offered in sensitivities from 10,50 , and $100 \mathrm{mV} / \mathrm{g}$, in one size, hermetically sealed, with dimensions as indicated on Figure 6.

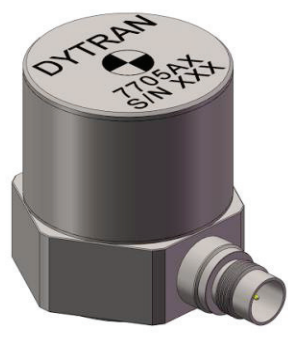

Fig. 4. Model of sensor 7705 A.

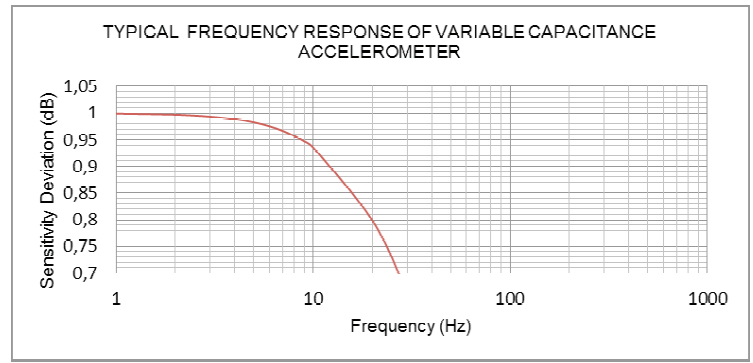

Fig. 1. Typical low frequency response of a piezoelectric accelerometer.

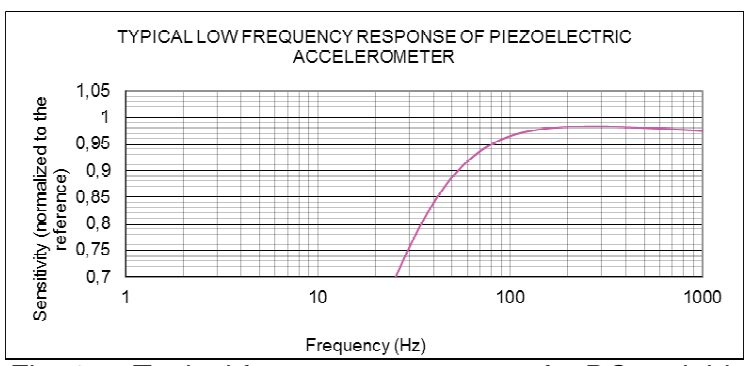

Fig. 2. Typical frequency response of a DC variable capacitance accelerometer.

The instrumentation amplifier performs a mathematical operation of subtraction on two input signals that are out of phase, and produces the continuous spectrum on the output:

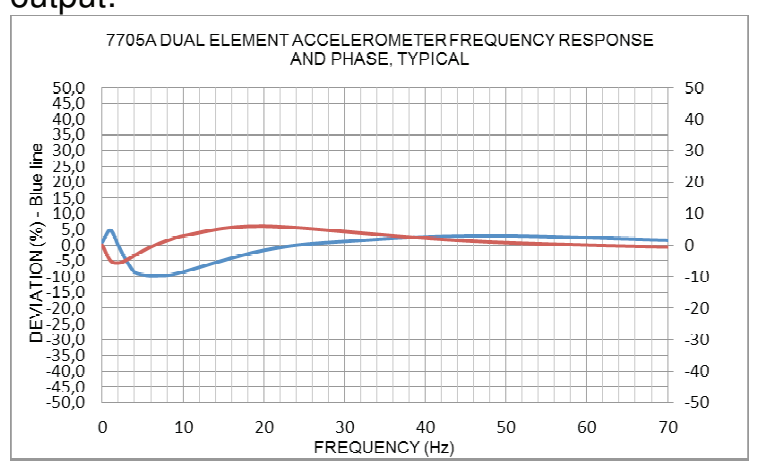

Fig. 2. Typical frequency responses of the instrumentation buffer output.

Due to slight imperfections of the filter geometries and a minor mismatch in sensitivities from variable capacitance to piezoelectric element the overlap point between two elements is designated by the visible ripple in the signal spectrum 
Frequency Response of Sensor

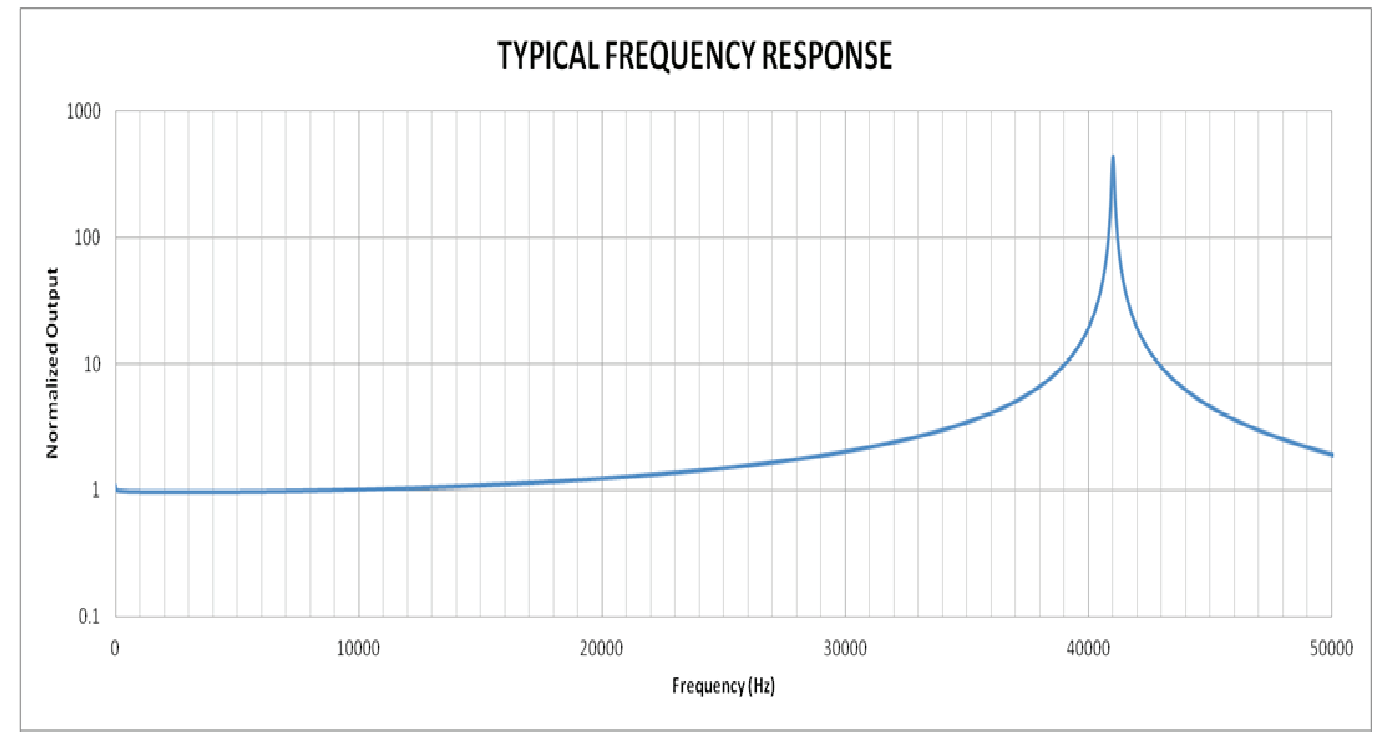

Fig. 5. Frequency response of the sensor $7705 A$..

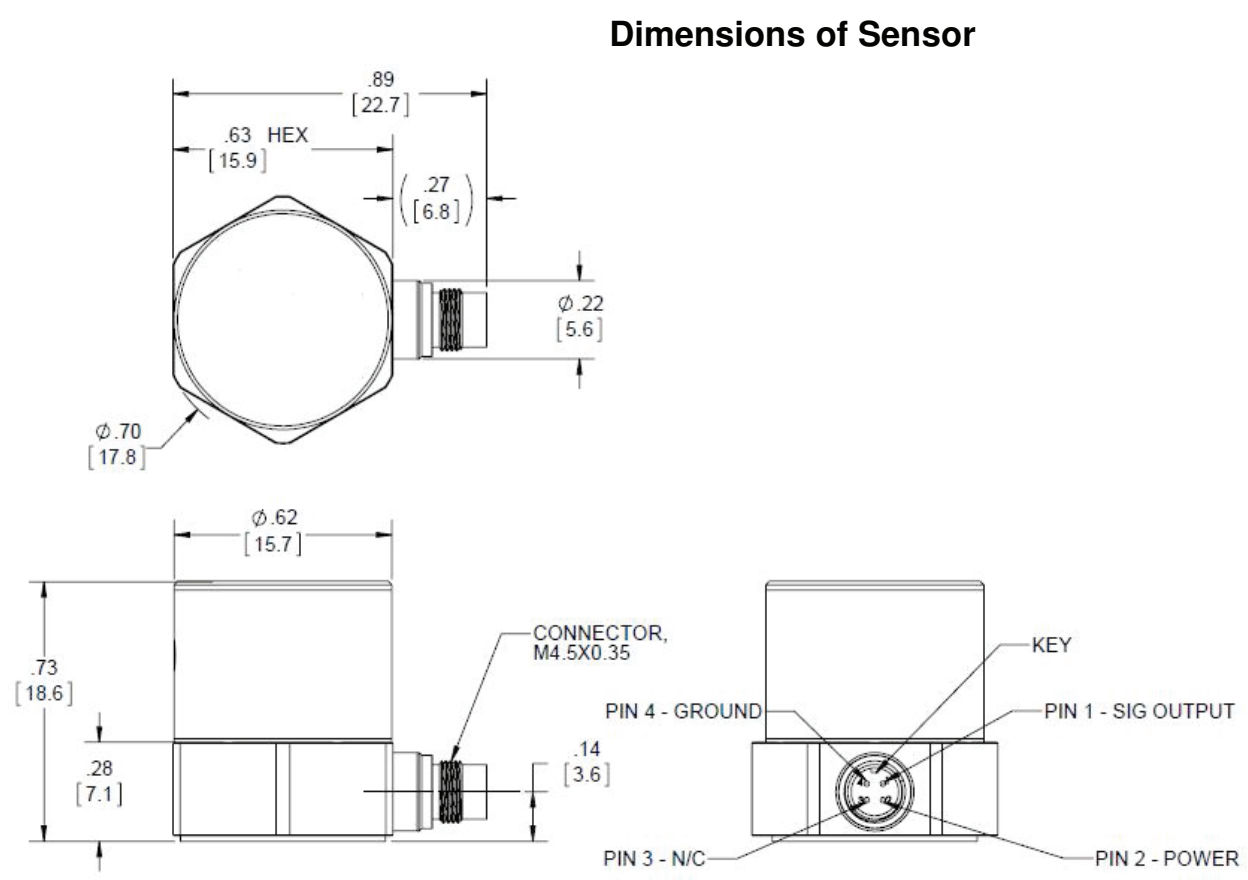

Fig. 5. Housing dimensions of model 7705A.

\section{Conclusion}

Requests from our customers have motivated Dytran to make sensors for measurement and monitoring for 34 years!

Dytran new series of accelerometers 7705A a.k.a ELF is a new generation of acceleration measurement instruments that combine two technologies which were used separately up to this moment. The accelerometer contains a piezoelectric and variable capacitance elements with respective filtering whose outputs are electrically superimposed over one another.

The result is an output from a single pin that provides one of the widest bandwidths from 0 $\mathrm{Hz}$ to $20 \mathrm{kHz}$ with great resolution of 0.0008 gRMS.

This is one of the innovations which have led to creating the smallest, lightest and hottest and coolest sensors in the world!

The best innovations are yet to come! 